\section{Properties of the Composite Quadratic Lyapunov Functions}

Tingshu $\mathrm{Hu}$ and Zongli Lin

\begin{abstract}
A composite quadratic Lyapunov function introduced recently was shown to be very useful in the study of set invariance properties for linear systems with input and state constraints and for systems with a class of convex/concave nonlinearities. In this note, more properties about this function are revealed. In particular, we study the continuity of the optimal parameter involved in this function. This continuity is crucial in the construction of a continuous feedback law which makes the convex hull of a group of ellipsoids invariant.
\end{abstract}

Index Terms-Convex hull, dual set, level set, Lyapunov function.

\section{INTRODUCTION}

Lyapunov functions play an important role in the study of nonlinear systems and the construction of Lyapunov functions is one of the most fundamental problems in systems theory. The most popular types of Lyapunov functions are the quadratic functions, piecewise-linear functions and piecewise-affine functions (see, e.g., [1], [2], and [15]).

For a general nonlinear system, there is no systematic method for constructing a Lyapunov function. If we restrict our attention to a certain category of nonlinear systems, we may have a better chance to identify a class of Lyapunov function candidates. For example, [13] constructed strict Lyapunov functions for periodic time-varying systems, [11], [12], [16] constructed Lyapunov functions for some cascaded nonlinear systems, and [10] proposed a class of piecewise quadratic functions for switched systems. More recently, we proposed a composite quadratic Lyapunov function for constrained control systems in [8]. We note that, both the piecewise quadratic function in [10] and the composite quadratic function in [8] are pertinent to quadratic functions and are generated by a set of positive definite matrices. As a result, the analysis problems in these papers were transformable into linear matrix inequalities (LMIs)

The composite quadratic Lyapunov function turned out to be very effective in dealing with constrained control systems as well as a class of more general nonlinear systems. For a constrained control system, a simple quadratic Lyapunov function could be a good candidate for local stability analysis and its level sets, the invariant ellipsoids could be used as estimates of the domain of attraction (see, e.g., [5], [7], and [18]). It is clear that the union of a group of invariant ellipsoids is also invariant. In [8], the composite quadratic Lyapunov function was introduced for further enlargement of the estimation of the domain of attraction. It was shown that, for a system under a given saturated linear feedback, the convex hull of a group of invariant ellipsoids is also invariant. Moreover, if we have a group of ellipsoids, each invariant under an individual saturated linear feedback, then a nonlinear feedback law can be constructed to make their convex hull invariant. In [6], the composite quadratic Lyapunov functions were used to study the invariance of the convex hull of ellipsoids for a class of nonlinear systems satisfying a generalized sector condition. The generalized sector is bounded by two symmetric functions which are concave or convex in the right-half plane.

Manuscript received April 6, 2003; revised March 10, 2004. Recommended by Associate Editor L. Glielmo. This work was supported in part by the National Science Foundation under Grant CMS-0324329.

The authors are with the Department of Electrical and Computer Engineering, University of Virginia, Charlottesville, VA 22904-4743 USA (e-mail: th7f,zl5y@virginia.edu).

Digital Object Identifier 10.1109/TAC.2004.831132
This note is intended to complement the results in [8]. We will reveal additional properties about the composite quadratic function and its level sets.

Notation

- For two integers $k_{1}, k_{2}, k_{1}<k_{2}$, we denote $I\left[k_{1}, k_{2}\right]=$ $k_{1}, k_{1}+1, \ldots, k_{2}$.

A symmetric positive-definite (semidefinite) matrix $P$ is denoted as $P>0(P \geq 0)$. For a $P \in \mathbf{R}^{n \times n}, P>0$, and a $\rho \in(0, \infty)$, denote $\mathcal{E}(P, \rho):=\left\{x \in \mathbf{R}^{n}: x^{\prime} P x \leq \rho\right\}$. For simplicity, we use $\mathcal{E}(P)$ to denote $\mathcal{E}(P, 1)$.

- For a vector $c \in \mathbf{R}^{n}$, define $\mathcal{L}(c):=\left\{x \in \mathbf{R}^{n}:\left|c^{\prime} x\right| \leq 1\right\}$.

- $\quad \Gamma_{N}:=\left\{\gamma \in \mathbf{R}^{N}: \sum_{j=1}^{N} \gamma_{j}=1, \gamma_{j} \geq 0\right\}$.

- For a set $S$ and a scalar $\alpha, \alpha S=\{\alpha x: x \in S\}$.

\section{COMPOSITE QUADRATIC FUNCTION AND SOME PRELIMINARIES}

\section{A. Composite Quadratic Function}

With a positive-definite matrix $P \in \mathbf{R}^{n \times n}$, a quadratic function can be defined as $V(x)=x^{\prime} P x$. For a positive number $\rho$, a level set of $V(x)$, denoted $L_{V}(\rho)$, is

$$
L_{V}(\rho):=\left\{x \in \mathbf{R}^{n}: V(x) \leq \rho\right\}=\mathcal{E}(P, \rho) .
$$

In [8], we introduced a function which is determined by a group of positive-definite matrices $P_{1}, P_{2}, \ldots, P_{N} \in \mathbf{R}^{n \times n}$. Let $Q_{j}=P_{j}^{-1}$, $j \in I[1, N]$. For a vector $\gamma \in \mathbf{R}^{N}$, define

$$
Q(\gamma):=\sum_{j=1}^{N} \gamma_{j} Q_{j} \quad P(\gamma):=Q^{-1}(\gamma) .
$$

Since $Q(\gamma), P(\gamma)>0$ for all $\gamma \in \Gamma_{N}$, these two matrix functions are analytic in $\gamma \in \Gamma_{N}$. The composite quadratic function is defined as

$$
V_{c}(x):=\min _{\gamma \in \Gamma_{N}} x^{\prime} P(\gamma) x .
$$

Clearly, $V_{c}(x)$ is a positive definite function. For $\rho>0$, the level set of $V_{c}(x)$ is

$$
L_{V_{c}}(\rho):=\left\{x \in \mathbf{R}^{n}: V_{c}(x) \leq \rho\right\} .
$$

Two useful properties of this composite quadratic function were presented in [8]. First, its level set is the convex hull of the level sets of $x^{\prime} P_{j} x$, the ellipsoids $\mathcal{E}\left(P_{j}, \rho\right), j \in I[1, N]$; Second, it is continuously differentiable with the partial derivative

$$
\frac{\partial V_{c}}{\partial x}=2 P\left(\gamma^{*}\right) x
$$

where $\gamma^{*}$ is an optimal $\gamma$ solving (1).

In [8], we applied the composite quadratic Lyapunov function in the study of the invariance of ellipsoids and their convex hull for the following constrained control system:

$$
\dot{x}=A x+B u
$$

where $x(t) \in X_{0} \subset \mathbf{R}^{n}, u(t) \in \mathbf{R}^{m}$, and $\left|u_{i}(t)\right| \leq 1$. One of the main results is that if each ellipsoid from a group $\mathcal{E}\left(P_{j}\right), j \in I[1, N]$, is invariant under the same saturated linear feedback law $u=\operatorname{sat}(F x)$ by certain condition, then the convex hull of these ellipsoids is also invariant. Another main result is that if each ellipsoid from a group $\mathcal{E}\left(P_{j}\right), j \in I[1, N]$, is invariant under a corresponding saturated linear feedback law $u=\operatorname{sat}\left(F_{j} x\right)$ by certain condition, then we can construct a nonlinear feedback law $u=F\left(\gamma^{*}(x)\right) x$ to make the convex hull of the ellipsoids invariant. It was established that the continuity of the 
new feedback law $u=F\left(\gamma^{*}(x)\right) x$ depends on the continuity of the function $\gamma^{*}(x)$. We are interested in the continuity of feedback laws not only because it facilitates implementation but also it is beneficial to the robustness of the system against sensor and actuator noise [17]. For this reason, the main task of this note is to derive conditions under which $\gamma^{*}(x)$ is continuous in $x$. We will also reveal some interesting properties about the function $V_{c}(x)$ and its level set.

\section{B. Some Facts About Convex Sets}

For easy reference, we collect in this section some results from convex analysis (e.g., see [4]).

The convex combination of $x_{1}, x_{2}, \ldots, x_{k} \in \mathbf{R}^{n}$ is an element of the form $\sum_{j=1}^{k} \gamma_{j} x_{j}, \gamma \in \Gamma_{k}$. A set $S$ is convex if and only if it contains every convex combination of its elements.

The convex hull of a set $S$, denoted $\operatorname{co}\{S\}$, is the set of all the convex combinations of the elements in $S$. If $S=\cup_{j=1}^{k} C_{j}$ and each $C_{j}$ is convex, then

$$
\operatorname{co}\{S\}=\left\{\sum_{j=1}^{k} \gamma_{j} x_{j}: \gamma \in \Gamma_{k}, x_{j} \in C_{j}, j \in I[1, k]\right\} .
$$

Let $S$ be a compact convex set.

- We say that $x_{0} \in S$ is an extreme point of $S$ if it cannot be represented as the convex combination of other points in $S$, i.e.,

$$
\begin{aligned}
& x_{0}=\sum_{i=1}^{k} \gamma_{i} x_{i}, \quad \gamma \in \Gamma_{k}, \text { if and only if } \\
& x_{1}=x_{2}=\cdots=x_{k}=x_{0} .
\end{aligned}
$$

- A hyperplane $c^{\prime} x=1$ is a supporting hyperplane of $S$ if $c^{\prime} x \leq 1, \forall x \in S$.

A hyperplane $c^{\prime} x=1$ is a supporting hyperplane of $S$ at $x_{0} \in \partial S$ if $c^{\prime} x \leq 1, \forall x \in S$ and $c^{\prime} x_{0}=1$. If $c^{\prime} x=1$ is a supporting hyperplane at $x_{0}$, then the vector $c$ is normal to $S$ at $x_{0}$, i.e., $c^{\prime}\left(x-x_{0}\right) \leq 0$ for all $x \in S$.

- The intersection of a supporting hyperplane with the set $S$ is called an exposed face of $S$. Every $x_{0} \in \partial S$ is in an exposed face. Let $x_{0}$ be an element of an exposed face $E$. If $x_{0}$ is represented as the convex combination of the elements of $S$, then these elements must all belong to $E$.

Fact 1: Let $S$ be a compact convex set. A point $x_{0}$ is an extreme point of $S$ if and only if it is an extreme point of any exposed face containing it.

This fact implies that, if $x_{0} \in S$ is not an extreme point, then it is not an extreme point of any exposed face. The following are some relations between an ellipsoid and a hyperplane.

Fact 2 [7]: For a vector $c \in \mathbf{R}^{n}$ and a matrix $P>0, \mathcal{E}(P) \subset \mathcal{L}(c)$ if and only if $c^{\prime} P^{-1} c \leq 1$.

1) The equality $c^{\prime} P^{-1} c=1$ holds if and only if the ellipsoid $\mathcal{E}(P)$ and the hyperplane $c^{\prime} x=1$ has a unique intersection at $x_{0}=$ $P^{-1} c$. In this case, $1=c^{\prime} x_{0}>c^{\prime} x \forall x \in \mathcal{E}(P) \backslash\left\{x_{0}\right\}$.

2) If $c^{\prime} P^{-1} c<1$, then the ellipsoid $\mathcal{E}(P)$ lies strictly between the hyperplanes $c^{\prime} x=1$ and $c^{\prime} x=-1$ without touching them.

\section{Properties of the Level Set and Its Dual Set}

It is easy to see that $V_{c}(k x)=k^{2} V_{c}(x)$. Hence, $L_{V_{c}}(\rho)=$ $\rho^{1 / 2} L_{V_{c}}(1)$. For this reason, we will be focused on $L_{V_{c}}(1)$ in this section.

Property 1: Any exposed face of $L_{V_{c}}(1)$ is a polygon with $N$ or less extreme points (vertices) which belongs to $\partial \mathcal{E}\left(P_{j}\right), j \in I[1, N]$.
Proof: Recall that $L_{V_{c}}(1)$ is the convex hull of $\mathcal{E}\left(P_{j}\right), j \in$ $I[1, N]$. First, we note that if

$$
x_{0} \in L_{V_{c}}(1) \backslash \cup_{j=1}^{N} \mathcal{E}\left(P_{j}\right)
$$

then $x_{0}$ can only be represented as the convex combination of at least two points in $\cup_{j=1}^{N} \mathcal{E}\left(P_{j}\right)$ and, hence, it is not an extreme point of $L_{V_{c}}(1)$. This implies that, if $x_{0}$ is an extreme point of $L_{V_{c}}(1)$, we must have $x_{0} \in \partial \mathcal{E}\left(P_{j}\right)$ for some $j$. (However, the converse is not necessarily true.)

Let $c^{\prime} x=1$ be a supporting hyperplane, then $c^{\prime} x \leq 1$ for all $x \in$ $L_{V_{c}}(1)$. Since $L_{V_{c}}(1)$ is symmetric, we must also have $c^{\prime} x \geq-1$ for all $x \in L_{V_{c}}(1)$. Hence $L_{V_{c}}(1) \subset \mathcal{L}(c)$ and $\mathcal{E}\left(P_{j}\right) \subset \mathcal{L}(c)$. Denote the intersection of $c^{\prime} x=1$ with $L_{V_{c}}(1)$ as $E$, then $E$ is an exposed face of $L_{V_{c}}(1)$. By Fact 1, any extreme point of $E$ must be an extreme point of $L_{V_{c}}(1)$, which belongs to $\partial \mathcal{E}\left(P_{j}\right)$ for some $j$. By Fact $2, c^{\prime} x=1$ has at most one intersection with each of $\partial \mathcal{E}\left(P_{j}\right)$, it follows that $E$ has at most $N$ extreme points which belong to $\partial \mathcal{E}\left(P_{j}\right), j \in I[1, N]$. Hence $E$ is a polygon with at most $N$ vertices.

Since $V_{c}(x)$ is positive definite, convex and $V_{c}(k x)=k^{2} V_{c}(x)$, we can use it to define a norm

$$
\|x\|_{c}:=V_{c}^{1 / 2}(x) .
$$

The dual set of $L_{V_{c}}(1)$ is defined as

$$
L_{V_{c}}^{*}(1):=\left\{s \in \mathbf{R}^{n}: s^{\prime} x \leq 1 \quad \forall x \in L_{V_{c}}(1)\right\} .
$$

Geometrically, $L_{V_{c}}^{*}(1)$ consists of the set of $s \in \mathbf{R}^{n}$ such that $L_{V_{c}}(1)$ lies between the two hyperplanes $s^{\prime} x=1$ and $s^{\prime} x=-1$, i.e., $L_{V_{c}}(1) \subset \mathcal{L}(s)$. Since $L_{V_{c}}(1)$ is the convex hull of $\mathcal{E}\left(P_{j}\right)$, $j \in I[1, N], L_{V_{c}}^{*}(1)$ consists of all the $s$ such that $\mathcal{E}\left(P_{j}\right) \subset \mathcal{L}(s)$ for all $j \in I[1, N]$. It follows from Fact 2 that $s \in L_{V_{c}}^{*}(1)$ if and only if

$$
s^{\prime} P_{j}^{-1} s \leq 1 \quad \forall j \in I[1, N] .
$$

Hence

$$
\begin{aligned}
L_{V_{c}}^{*}(1) & =\left\{s \in \mathbf{R}^{n}: s^{\prime} P_{j}^{-1} s \leq 1, j \in I[1, N]\right\} \\
& =\cap_{j=1}^{N} \mathcal{E}\left(P_{j}^{-1}\right) .
\end{aligned}
$$

In summary, we have the following.

Property 2: The dual set of $L_{V_{c}}(1)$ is $\cap_{j=1}^{N} \mathcal{E}\left(P_{j}^{-1}\right)$.

The dual norm of $\|\cdot\|_{c}$ is defined as

$$
\|s\|_{c}^{*}:=\max \left\{s^{\prime} x:\|x\|_{c} \leq 1\right\}=\max \left\{s^{\prime} x: x \in L_{V_{c}}(1)\right\} .
$$

It is clear from (3) and (4) that

$$
L_{V_{c}}^{*}(1)=\left\{s \in \mathbf{R}^{n}:\|s\|_{c}^{*} \leq 1\right\} .
$$

By duality

$$
\begin{aligned}
\|x\|_{c} & =\max \left\{s^{\prime} x: s \in L_{V_{c}}^{*}(1)\right\} \\
& =\max \left\{s^{\prime} x: s^{\prime} P_{j}^{-1} s \leq 1, j \in I[1, N]\right\} .
\end{aligned}
$$

This implies that we have another way to compute $V_{c}(x)$, i.e.,

$$
V_{c}^{1 / 2}(x)=\max \left\{s^{\prime} x: s^{\prime} P_{j}^{-1} s \leq 1, j \in I[1, N]\right\} .
$$

As compared to the original definition of $V_{c}(x)$ in (1), the computation of $V_{c}(x)$ via (5) can be interpreted geometrically: $V_{c}^{1 / 2}(x)$ is the maximal value of $\alpha$ such that the hyperplane $x^{\prime} s=\alpha$ (in the $s$-space) touches the intersection of a group of ellipsoids, $\cap_{j=1}^{N} \mathcal{E}\left(P_{j}^{-1}\right)$.

With the dual norm, a conjugate Lyapunov function can be derived. It turns out that conjugate Lyapunov functions can be used to enhance stability and performance analysis of linear differential inclusions and 
nonlinear systems. Further results about the dual relationships between the composite quadratic Lyapunov function and its conjugate, along with the applications, have been developed in [3] and [9].

\section{PROPERTIES OF THE Optimization PROBLEM}

The optimization problem in (1) can be written as

$$
\begin{array}{r}
V_{c}(x)=\min x^{\prime} P(\gamma) x=\min x^{\prime}\left(\sum_{j=1}^{N} \gamma_{j} Q_{j}\right)^{-1} x \\
\text { s.t. } \sum_{j=1}^{N} \gamma_{j}=1 \quad \gamma_{j} \geq 0, j \in I[1, N] .
\end{array}
$$

In this section, we investigate the continuity of the optimal $\gamma$ as a function of $x$.

Proposition 1: For a given $x_{0} \in \mathbf{R}^{n}$, let $\gamma^{*} \in \Gamma_{N}$ be an optimal $\gamma$ such that

$$
x_{0}^{\prime} P\left(\gamma^{*}\right) x_{0}=\min _{\gamma \in \Gamma_{N}} x_{0}^{\prime} P(\gamma) x_{0}=V_{c}\left(x_{0}\right)
$$

Denote $x_{j}=Q_{j} P\left(\gamma^{*}\right) x_{0}$. Then

$$
x_{0}=\sum_{j=1}^{N} \gamma_{j}^{*} x_{j}
$$

i.e., $x_{0}$ is a convex combination of $x_{j}, j \in I[1, N]$. Let $h=P\left(\gamma^{*}\right) x_{0}$ and $\rho=V_{c}\left(x_{0}\right)$. If $\gamma_{j}^{*}>0$, then $x_{j} \in \partial \mathcal{E}\left(P_{j}, \rho\right)$. Moreover, $x_{j}$ and $x_{0}$ are in the same supporting hyperplane $h^{\prime} x=\rho$.

Proof: To characterize the property of the optimal solutions, let us introduce auxiliary parameters $r_{j}, j \in I[1, N]$ such that $\gamma_{j}-r_{j}^{2}=$ 0 , and $\alpha, \beta_{j}, j \in I[1, N]$. Define the Lagrangian as

$$
\begin{aligned}
L(\gamma, r, \alpha, \beta):=x_{0}^{\prime}\left(\sum_{j=1}^{N} \gamma_{j} Q_{j}\right)^{-1} & x_{0} \\
& +\alpha\left(\sum_{j=1}^{N} \gamma_{j}-1\right)+\sum_{j=1}^{N} \beta_{j}\left(\gamma_{j}-r_{j}^{2}\right) .
\end{aligned}
$$

The following is the set of conditions for a stationary point, which must be satisfied by an optimal solution $\left(\gamma^{*}, r^{*}, \alpha^{*}, \beta^{*}\right)$

$$
\begin{aligned}
& x_{0}^{\prime}\left(\sum_{j=1}^{N} \gamma_{j}^{*} Q_{j}\right)^{-1} Q_{j}\left(\sum_{j=1}^{N} \gamma_{j}^{*} Q_{j}\right)^{-1} x_{0} \\
& \quad-\alpha^{*}-\beta_{j}^{*}=0, \quad j \in I[1, N] \quad\left(\text { w.r.t } \gamma_{j}\right) \\
& \left.\sum_{j=1}^{N} \gamma_{j}^{*}=1 \quad \text { (w.r.t } \alpha\right) \\
& \left.\gamma_{j}^{*}=\left(r_{j}^{*}\right)^{2}, \quad j \in I[1, N] \quad \text { (w.r.t } \beta_{j}\right) \\
& \left.\beta_{j}^{*} r_{j}^{*}=0, \quad j \in I[1, N] \quad \text { (w.r.t } r_{j}\right) .
\end{aligned}
$$

Without loss of generality, assume that $\gamma_{j}^{*}>0$ for $j \in I\left[1, N_{0}\right]$, and $\gamma_{j}^{*}=0$ for $j \in I\left[N_{0}+1, N\right]$. Then, $r_{j}^{*} \neq 0$ for $j \in I\left[1, N_{0}\right]$, and $r_{j}^{*}=0$ for $j \in I\left[N_{0}+1, N\right]$. From (11), we have $\beta_{j}^{*}=0$ for $j \in I\left[1, N_{0}\right]$. It follows from (8) that

$$
\begin{aligned}
x_{0}^{\prime}\left(\sum_{j=1}^{N} \gamma_{j}^{*} Q_{j}\right)^{-1} Q_{j}\left(\sum_{j=1}^{N} \gamma_{j}^{*} Q_{j}\right)^{-1} x_{0}=\alpha^{*} \\
\forall j \in I\left[1, N_{0}\right] .
\end{aligned}
$$

Multiplying both sides with $\gamma_{j}^{*}$ and summing up the equalities for all $j \in I\left[1, N_{0}\right]$, we obtain

$$
\begin{aligned}
x_{0}^{\prime}\left(\sum_{j=1}^{N} \gamma_{j}^{*} Q_{j}\right)^{-1} & \left(\gamma_{1}^{*} Q_{1}+\cdots+\gamma_{N_{0}}^{*} Q_{N_{0}}\right) \\
& \cdot\left(\sum_{j=1}^{N} \gamma_{j}^{*} Q_{j}\right)^{-1} x_{0}=\alpha^{*}\left(\gamma_{1}^{*}+\cdots+\gamma_{N_{0}}^{*}\right)=\alpha^{*} .
\end{aligned}
$$

Noticing that $\gamma_{j}^{*}=0$ for $j \in I\left[N_{0}+1, N\right]$. Hence, we have

$$
V_{c}\left(x_{0}\right)=x_{0}^{\prime}\left(\sum_{j=1}^{N} \gamma_{j}^{*} Q_{j}\right)^{-1} x_{0}=\alpha^{*} .
$$

Let $\rho=V_{c}\left(x_{0}\right)=\alpha^{*}$ and let $x_{j}=Q_{j} P\left(\gamma^{*}\right) x_{0}=$ $Q_{j}\left(\sum_{j=1}^{N} \gamma_{j}^{*} Q_{j}\right)^{-1} x_{0}$, then it follows from (8) that for all $j \in I\left[1, N_{0}\right]$

$$
\begin{aligned}
x_{j}^{\prime} P_{j} x_{j} & =x_{0}^{\prime}\left(\sum_{j=1}^{N} \gamma_{j}^{*} Q_{j}\right)^{-1} Q_{j}\left(\sum_{j=1}^{N} \gamma_{j}^{*} Q_{j}\right)^{-1} x_{0} \\
& =\rho \Rightarrow x_{j} \in \partial \mathcal{E}\left(P_{j}, \rho\right)
\end{aligned}
$$

and

$$
\begin{aligned}
\sum_{j=1}^{N_{0}} \gamma_{j}^{*} x_{j} & =\sum_{j=1}^{N} \gamma_{j}^{*} x_{j} \\
& =\left(\sum_{j=1}^{N} \gamma_{j}^{*} Q_{j}\right)\left(\sum_{j=1}^{N} \gamma_{j}^{*} Q_{j}\right)^{-1} x_{0}=x_{0} .
\end{aligned}
$$

This proves (7), which means that $x_{0}$ is the convex combination of $x_{j} \in \partial \mathcal{E}\left(P_{j}, \rho\right), j \in I\left[1, N_{0}\right]$. Moreover

$$
P_{j} x_{j}=\left(\sum_{j=1}^{N} \gamma_{j}^{*} Q_{j}\right)^{-1} x_{0}=P\left(\gamma^{*}\right) x_{0} .
$$

If we let $h=P\left(\gamma^{*}\right) x_{0}=P_{j} x_{j}$, then from (2), we know that $h$ is normal to the set $L_{V_{c}}(\rho)$ at $x_{0}$ and the hyperplane $h^{\prime} x=\rho\left(=h^{\prime} x_{0}\right)$ is a supporting hyperplane at $x_{0}$. Moreover, for $j=1,2, \ldots, N_{0}$

$$
h^{\prime} x_{j}=x_{j}^{\prime} P_{j} x_{j}=\rho .
$$

This shows that $x_{0}$ and $x_{j}, j \in I\left[1, N_{0}\right]$, are on the same supporting hyperplane $h^{\prime} x=\rho$.

From Proposition 1, we see that an $x_{0} \in \partial L_{V_{c}}(\rho)$ can be represented as a convex combination of $x_{j} \in \partial \mathcal{E}\left(P_{j}, \rho\right), j \in I[1, N]$ in terms of an optimal $\gamma^{*}$. (If $\gamma_{j}^{*}=0$, then the corresponding $x_{j}$ can be any point in $\left.\partial \mathcal{E}\left(P_{j}\right)\right)$. As we have said, it is desirable that $\gamma^{*}$ is continuous in $x$. It turns out that this continuity depends on the uniqueness of the optimal $\gamma^{*}$.

Proposition 2:

1) If for each $x \in \partial L_{V_{c}}(1)$, there exists a unique representation

$$
x=\sum_{j=1}^{N} \gamma_{j} x_{j}, \quad x_{j} \in \partial \mathcal{E}\left(P_{j}\right), \quad \gamma \in \Gamma_{N}
$$

then $\gamma^{*}(x)$ is unique for every $x \in \mathbf{R}^{n}$.

2) If $\gamma^{*}(x)$ is unique for every $x \in \mathbf{R}^{n}$, then it is continuous in $x$. Proof:

1) If $\gamma^{*}$ is not unique for a certain $x_{0}$, then by Proposition 1, there exist different representations of $x_{0}$ in terms of the different $\gamma^{*}$ 's.

2) To prove the continuity, we show that if $\gamma^{*}(x)$ is not continuous at certain $x_{0}$, then there exist representations of $x_{0}$ in terms of different $\gamma^{*}$,s. 
Consider $x_{0} \in \partial L_{V_{c}}(1)$. Suppose on the contrary that $\gamma^{*}(x)$ is not continuous at $x_{0}$. Then there exists a sequence $\left\{x^{k}\right\}$ such that $\lim _{k \rightarrow \infty} x^{k}=x_{0}$, and $\gamma^{*}\left(x^{k}\right)$ does not converge to $\gamma^{*}\left(x_{0}\right)$. Since $\gamma^{*}\left(x^{k}\right) \in \Gamma_{N}$ and $\Gamma_{N}$ is a compact set, there exists a subsequence of $\left\{x^{k}\right\},\left\{x^{1 k}\right\}$, and a constant vector $\gamma^{* 1} \in \Gamma_{N}$ such that

$$
\lim _{k \rightarrow \infty} \gamma^{*}\left(x^{1 k}\right)=\gamma^{* 1} \neq \gamma^{*}\left(x_{0}\right) \text {. }
$$

For simplicity, let $\gamma^{* 1 k}=\gamma^{*}\left(x^{1 k}\right)$. Then, by Proposition 1, there exist $x_{j}^{1 k} \in \partial \mathcal{E}\left(P_{j}\right), j \in I[1, N], k \in I[1, \infty]$ such that

$$
x^{1 k}=\sum_{j=1}^{N} \gamma_{j}^{* 1 k} x_{j}^{1 k} .
$$

Since the sequence $\left\{\left(\gamma^{* 1 k}, x^{1 k}, x_{1}^{1 k}, \ldots, x_{N}^{1 k}\right)\right\}$ is in a compact set, there exists a subsequence of it, $\left\{\left(\gamma^{* 2 k}, x^{2 k}, x_{1}^{2 k}, \ldots, x_{N}^{2 k}\right)\right\}$, such that

$$
\lim _{k \rightarrow \infty} x_{j}^{2 k}=x_{j}^{2}, \quad j \in I[1, N] .
$$

Since $\left\{\left(\gamma^{* 2 k}, x^{2 k}, x_{1}^{2 k}, \ldots, x_{N}^{2 k}\right)\right\}$ is a subsequence of $\left\{\left(\gamma^{* 1 k}, x^{1 k}, x_{1}^{1 k}, \ldots, x_{N}^{1 k}\right)\right\}$, from (13), we have

$$
x^{2 k}=\sum_{j=1}^{N} \gamma_{j}^{* 2 k} x_{j}^{2 k} .
$$

We also have

$$
\lim _{k \rightarrow \infty} \gamma^{* 2 k}=\lim _{k \rightarrow \infty} \gamma^{* 1 k}=\gamma^{* 1}
$$

and

$$
\lim _{k \rightarrow \infty} x^{2 k}=\lim _{k \rightarrow \infty} x^{1 k}=x_{0}
$$

Therefore

$$
x_{0}=\sum_{j=1}^{N} \gamma_{j}^{* 1} x_{j}^{2} .
$$

By Proposition $1, x_{0}$ can also be represented as

$$
x_{0}=\sum_{j=1}^{N} \gamma_{j}^{*}\left(x_{0}\right) x_{j}
$$

for some $x_{j} \in \partial \mathcal{E}\left(P_{j}\right), j \in I[1, N]$. Since $\gamma^{* 1} \neq \gamma^{*}\left(x_{0}\right)$, (14) and (15) mean that $x_{0}$ has two different representations. This is a contradiction. Hence $\gamma^{*}(x)$ must be continuous at any $x_{0} \in \partial L_{V_{c}}(1)$. The continuity of $\gamma^{*}(x)$ in the whole space follows from the fact that $\gamma^{*}(\alpha x)=\gamma^{*}(x)$ for every $\alpha \in \mathbf{R}$.

We now consider the condition in Proposition 2, the uniqueness of the representation of a point $x \in \partial L_{V_{c}}(1)$. Any point $x \in \partial L_{V_{c}}(1)$ belongs to an exposed face of $L_{V_{c}}(1)$, which is the intersection of $\partial L_{V_{c}}(1)$ with a certain supporting hyperplane. By Property 1 , we know that an exposed face is a polygon whose vertices belong to $\partial \mathcal{E}\left(P_{j}\right)$ ), $j \in I[1, N]$.

We also know that, to express $x_{0} \in \partial L_{V_{c}}(1)$ as the convex combination of the elements of $L_{V_{c}}(1)$, these elements must be in the same exposed face with $x_{0}$. Hence, we only need to consider the uniqueness of representing $x_{0}$ as the convex combination of those $x_{j} \in \partial \mathcal{E}\left(P_{j}\right)$ which are in the same exposed face with $x_{0}$.

Recall that a supporting hyperplane has at most one intersection with an ellipsoid $\mathcal{E}\left(P_{j}\right)$. Without loss of generality, suppose that an exposed face $E$ has intersections with the first $N_{0}$ ellipsoids, and the intersections are $x_{j} \in \partial \mathcal{E}\left(P_{j}\right), j \in I\left[1, N_{0}\right]$. From Property 1 , we know that the vertices of $E$ belong to $\left\{x_{j}: j \in I\left[1, N_{0}\right]\right\}$. Consider a point $x_{0} \in E$. Then, $x_{0}$ can only be represented as a convex combination of $x_{j}, j \in I\left[1, N_{0}\right]$. The condition for the uniqueness of such a representation is stated as follows.

Proposition 3: Let $E$ be an exposed face of $L_{V_{c}}(1)$ which has intersections with $N_{0}$ ellipsoids, $x_{j} \in \partial \mathcal{E}\left(P_{j}\right), j \in I\left[1, N_{0}\right]$. Then every $x_{0} \in E$ can be uniquely represented as a convex combination of $x_{j}$, $j \in I\left[1, N_{0}\right]$, if and only if the vectors $x_{j}, j \in I\left[1, N_{0}\right]$, are linearly independent.

Proof: Suppose that there exists an $x_{0} \in E$ whose representation is not unique, i.e., there exist different $\alpha, \beta \in \Gamma_{N}$, such that

$$
x_{0}=\sum_{j=1}^{N_{0}} \alpha_{j} x_{j}=\sum_{j=1}^{N_{0}} \beta_{j} x_{j}
$$

then

$$
\sum_{j=1}^{N_{0}}\left(\alpha_{j}-\beta_{j}\right) x_{j}=0
$$

which implies that $x_{j}, j \in I\left[1, N_{0}\right]$ are linearly dependent.

We next show that linear dependence of $x_{j}$ 's implies nonuniqueness. Recall that $x_{j}$ 's are in the same hyperplane, i.e., there exists a vector $h \in \mathbf{R}^{n}$ such that

$$
h^{\prime} x_{j}=1, \quad j \in I\left[1, N_{0}\right] .
$$

Suppose that $x_{j}$ 's are linearly dependent, then there exists $\alpha_{j}, j \in$ $I\left[1, N_{0}\right]$ such that

$$
\sum_{j=1}^{N_{0}} \alpha_{j} x_{j}=0 .
$$

Multiplying both sides with $h^{\prime}$ from left and using (16), we obtain

$$
\sum_{j=1}^{N_{0}} \alpha_{j}=0 .
$$

This means that the sum of the positive $\alpha_{j}$ 's equals to the absolute value of the sum of the negative $\alpha_{j}$ 's. Without loss of generality, assume that $\alpha_{j}>0$ for $j \in I\left[1, N_{1}\right]$ and $\alpha_{j} \leq 0$ for $j \in I\left[N_{1}+1, N_{0}\right]$, then we have

$$
\sum_{j=1}^{N_{1}} \alpha_{j}=-\sum_{j=N_{1}+1}^{N_{0}} \alpha_{j} .
$$

From (17) and the previous equation, we obtain

$$
\frac{1}{\sum_{j=1}^{N_{1}} \alpha_{j}} \sum_{j=1}^{N_{1}} \alpha_{j} x_{j}=\frac{1}{\sum_{j=N_{1}+1}^{N_{0}} \alpha_{j}} \sum_{j=N_{1}+1}^{N_{0}} \alpha_{j} x_{j} .
$$

This shows that the point

$$
x_{0}=\frac{1}{\sum_{j=1}^{N_{1}} \alpha_{j}} \sum_{j=1}^{N_{1}} \alpha_{j} x_{j} \in E
$$

has nonunique representations.

We now interpret the condition in Proposition 3. There are two cases where $x_{j}$ 's, the intersections of $E$ with the ellipsoids, are linearly dependent.

Case 1) $N_{0}$, the number of intersections, is greater than $n$, the dimension of the state space.

Case 2) $N_{0} \leq n$ but $x_{j}$ 's are still dependent.

Actually, given a group of randomly chosen ellipsoids, the probability that the above situations will occur is 0 . We first explain the first case. For simplicity, assume that there exists a hyperplane which has a unique intersection with each ellipsoid in a group of $n+1$. From Fact 2, a hyperplane $c^{\prime} x=1$ has a unique intersection with an ellipsoid $\mathcal{E}\left(P_{j}\right)$ if and only if $c^{\prime} P_{j}^{-1} c=1$. Hence, we have the following.

Proposition 4: Let $\mathcal{E}\left(P_{j_{1}}\right), \mathcal{E}\left(P_{j_{2}}\right), \ldots, \mathcal{E}\left(P_{j_{(n+1)}}\right)$ be a selection of $n+1$ ellipsoids from the original $N$ ellipsoids. There exists 
a hyperplane which has a unique intersection with each of these ellipsoids if and only if there exists a vector $c \in \mathbf{R}^{n}$ such that

$$
c^{\prime} P_{j_{1}}^{-1} c=c^{\prime} P_{j_{2}}^{-1} c=\cdots=c^{\prime} P_{j_{(n+1)}}^{-1} c=1 .
$$

There are $n+1$ equations in (18) and there are only $n$ unknown variables. Generally, such equation systems have no solution. That is, given randomly chosen $n+1$ ellipsoids in $\mathbf{R}^{n}$, the probability that all of their boundaries intersect at one point is 0 . This can be easily visualized for $n=2$. If the first two ellipsoid boundaries have four intersections, the probability that the third ellipsoid boundary will include one of these four points is zero. In short, Case 1) occurs with a zero probability.

Similarly, Case 2) can be characterized as the existence of $j_{1}, j_{2}, \ldots, j_{N_{0}} \in I[1, N]$, and a vector $c \in \mathbf{R}^{n}$ such that

$$
c^{\prime} P_{j_{1}}^{-1} c=c^{\prime} P_{j_{2}}^{-1} c=\cdots=c^{\prime} P_{j_{N_{0}}}^{-1} c=1, \quad N_{0} \leq n
$$

and $P_{j_{1}}^{-1} c, P_{j_{2}}^{-1} c, \ldots$, and $P_{j_{N_{0}}}^{-1} c$ are linearly dependent, i.e., there exists $\alpha_{i} \in \mathbf{R}, i \in I\left[1, N_{0}\right]$, such that

$$
\sum_{i=1}^{N_{0}} \alpha_{i} P_{j_{i}}^{-1} c=0 \quad \sum_{i=1}^{N_{0}} \alpha_{i}^{2}=1 .
$$

We note here that $P_{j_{i}}^{-1} c, i \in I\left[1, N_{0}\right]$ are the intersections of the supporting hyperplane $c^{\prime} x=1$ with the ellipsoids. In the general case, the system of equations in (19) and (20) have no solution since there are $N_{0}+n+1$ scalar equations and only $N_{0}+n$ unknowns. Hence, Case 2) also occurs with a zero probability.

Combining Propositions 2-4 and the foregoing discussion, we obtain the following.

Theorem 1: If none of the following is true, then $\gamma^{*}(x)$ is continuous and

1) there exists $c \in \mathbf{R}^{n}$ and $j_{1}, j_{2}, \ldots, j_{n+1} \in I[1, N]$ satisfying (18);

2) there exists $c \in \mathbf{R}^{n}, j_{1}, j_{2}, \ldots, j_{N_{0}} \in I[1, N]$ and $\alpha_{i}, i \in$ $I\left[1, N_{0}\right]$ satisfying (19) and (20).

With a finite number of ellipsoids, there are finite number of combinations of equations in (18) and (19) [coupled with (20)]. Since both of them are satisfied with a zero probability, the function $\gamma^{*}(x)$ is generally continuous.

The conditions in Theorem 1 can be verified by numerical methods. Actually, both of these conditions involve solving multivariate polynomial equations, for which efficient algorithms can be found, e.g., in [14]. Our polynomials in (18)-(20) are all of quadratic forms. By using this property and the algorithm in [14], we have developed a simple algorithm to compute all the solutions for a system of three quadratic polynomial equations in three variables. Here, we use an example to illustrate the main result of this section.

Example 1: We randomly generated four positive-definite matrices

$$
\begin{aligned}
Q_{1} & =\left[\begin{array}{ccc}
0.4480 & -0.0699 & 0.0865 \\
-0.0699 & 0.4958 & 0.4780 \\
0.0865 & 0.4780 & 0.5461
\end{array}\right] \\
Q_{2} & =\left[\begin{array}{ccc}
0.2347 & 0.0641 & 0.1653 \\
0.0641 & 0.4992 & -0.4691 \\
0.1653 & -0.4691 & 0.7223
\end{array}\right] \\
Q_{3} & =\left[\begin{array}{ccc}
0.8436 & -0.0018 & 0.2561 \\
-0.0018 & 0.1751 & -0.0132 \\
0.2561 & -0.0132 & 0.3984
\end{array}\right] \\
Q_{4} & =\left[\begin{array}{ccc}
0.7272 & -0.3058 & -0.1936 \\
-0.3058 & 0.5732 & 0.4212 \\
-0.1936 & 0.4212 & 0.4652
\end{array}\right] .
\end{aligned}
$$

Let $P_{i}=Q_{i}^{-1}, i=1,2,3,4$. We would like to check the continuity of $\gamma^{*}(x)$ by Theorem 1 . For item 1), we need to check if

$$
c^{\prime} Q_{1} c=c^{\prime} Q_{2} c=c^{\prime} Q_{3} c=c^{\prime} Q_{4} c=1
$$

has a solution. We find all eight solutions for $c^{\prime} Q_{1} c=c^{\prime} Q_{2} c=$ $c^{\prime} Q_{3} c=1$ and none of them satisfies $c^{\prime} Q_{4} c=1$. Since the solutions are symmetric, we list four of them and the corresponding values of $c^{\prime} Q_{4} c$ as follows:

$$
\begin{array}{rlrl}
c_{1}^{\prime} & =\left[\begin{array}{lll}
0.5274 & 0.1250 & 1.0888
\end{array}\right], & c_{1}^{\prime} Q_{4} c_{1}=0.6147 \\
c_{2}^{\prime}=\left[\begin{array}{lll}
1.1657 & 0.1031 & -1.1766
\end{array}\right], & c_{2}^{\prime} Q_{4} c_{2}=1.9937 \\
c_{3}^{\prime}=\left[\begin{array}{lll}
0.8838 & -1.1576 & 0.1845
\end{array}\right], & c_{3}^{\prime} Q_{4} c_{3}=1.7347 \\
c_{4}^{\prime}=\left[\begin{array}{lll}
0.9327 & 1.1811 & 0.0550
\end{array}\right], & c_{4}^{\prime} Q_{4} c_{4}=0.7948 .
\end{array}
$$

Hence, we conclude that (21) has no solution.

We next proceed to check item 2). For $N_{0}=3$, we find the solutions to $c^{\prime} Q_{j_{1}} c=c^{\prime} Q_{j_{2}} c=c^{\prime} Q_{j_{3}} c=1$ for all the possible combinations $j_{1}, j_{2}, j_{3}$ and check each solution to see if the least singular value of the matrix $\left[\begin{array}{llll}Q_{j_{1}} c & Q_{j_{2}} c & Q_{j_{3}} c\end{array}\right]$ is less than a threshold value, e.g., 0.0001 . For the combination $\left(j_{1}, j_{2}, j_{3}\right)=(1,2,3)$, the solutions are $\pm c_{i}, i=1,2,3,4$, and the least singular values of the matrix for $c_{i}$ 's are $0.3653,0.4637,0.4756,0.4807$, respectively. We do the same for other three combinations of $\left(j_{1}, j_{2}, j_{3}\right)$ and find that the least singular values are all much greater than the threshold value.

For $N_{0}=2$, the existence of solutions satisfying (19) and (20) implies that $Q_{j_{1}}-Q_{j_{2}}$ is singular, which is not the case for any combination of $j_{1}$ and $j_{2}$.

In summary, there exists no vector $c$ satisfying either item 1) or 2) of Theorem 1 . Hence we conclude that the function $\gamma^{*}(x)$ is continuous for this set of $\left(Q_{1}, Q_{2}, Q_{3}, Q_{4}\right)$.

We designed a test to check the probability that item 1 ) of Theorem 1 is nearly satisfied. We generated 10000 sets of $\left(Q_{1}, Q_{2}, Q_{3}, Q_{4}\right)$. For each of the set we solve for $c$ such that $c^{\prime} Q_{1} c=c^{\prime} Q_{2} c=c^{\prime} Q_{3} c=1$ and check the value of $c^{\prime} Q_{4} c$. For a given $\varepsilon<1$, we count the number of sets where one of the solutions satisfies $\left|c^{\prime} Q_{4} c-1\right| \leq \varepsilon$. The result is: There are 231 sets for $\varepsilon=0.01,28$ for $\varepsilon=0.001,2$ for $\varepsilon=0.0001$ and none for $\varepsilon=0.00001$.

\section{CONCLUSION}

We presented several important properties about the composite quadratic function and its level set. We showed that the exposed faces of the level set are polygons and its dual is the intersection of a group of ellipsoids. We also studied in detail the solution to the optimization problem in the definition of the composite quadratic function. A condition was derived for the continuity of an optimal parameter $\gamma$, which is crucial to the construction of continuous feedback laws that make the convex hull of a group of ellipsoids invariant. This condition for continuity can be verified by solving systems of polynomial equations.

\section{REFERENCES}

[1] F. Blanchini, "Set invariance in control-A survey," Automatica, vol. 35, pp. 1747-1767, 1999.

[2] G. Ferrari-Trecate, F. A. Cuzzola, D. Mignone, and M. Morari, "Analysis of discrete-time piecewise affine and hybrid systems," Automatica, vol. 38, pp. 2139-2146, 2002.

[3] R. Goebel, A. R. Teel, T. Hu, and Z. Lin, "Dissipativity for dual linear differential inclusions through conjugate storage functions," in IEEE Conf. Decision Control, 2004, submitted for publication.

[4] J.-B. Hiriart-Urruty and C. Lemarèchal, Convex Analysis and Minimization Algorithms I. New York: Springer-Verlag, 1993.

[5] H. Hindi and S. Boyd, "Analysis of linear systems with saturating using convex optimization," in Proc. 37th IEEE Conf. Decision Control, 1998, pp. 903-908.

[6] T. Hu, B. Huang, and Z. Lin, "Absolute stability with generalized sector condition,” IEEE Trans. Automat. Contr., vol. 49, pp. 535-548, Apr. 2004. 
[7] T. Hu and Z. Lin, Control Systems With Actuator Saturation. Boston, MA: Birkhäuser, 2001.

[8] — - "Composite quadratic Lyapunov functions for constrained control systems," IEEE Trans. Automat. Contr., vol. 48, pp. 440-450, Mar. 2003.

[9] T. Hu, Z. Lin, R. Goebel, and A. R. Teel, "Stability regions for saturated linear systems via conjugate Lyapnov functions," in IEEE Conf. Decision Control, 2004, submitted for publication.

[10] M. Johansson and A. Rantzer, "Computation of piecewise quadratic Lyapunov functions for hybrid systems," IEEE Trans. Automat. Contr., vol. 43, pp. 555-559, May 1998.

[11] R. Lozano, B. Brogliato, and I. D. Landau, "Passitivity and global stabilization of cascaded nonlinear systems," IEEE Trans. Automat. Contr., vol. 37, pp. 1386-1388, Sept. 1992.

[12] F. Mazenc, R. Sepulchre, and M. Jankovic, "Lyapunov functions for stable cascades and applications to global stabilization," IEEE Trans. Automat. Contr., vol. 44, pp. 1795-1800, Nov. 1999.

[13] F. Mazenc, "Strict Lyapunov functions for time-varying systems," $A u$ tomatica, vol. 39, pp. 349-353, 2003.

[14] H. M. Moller and R. Tenberg, "Multivariate polynomial system solving using intersections of eigenspaces," J. Symb. Comput., vol. 32, pp. 513-531, 2001.

[15] B. E. A. Milani, "Piecewise-affine Lyapunov functions for discrete-time linear systems with saturating controls," in Proc. Amer. Control Conf., 2001, pp. 4206-4211.

[16] L. Praly, B. d'Andrea-Novel, and J.-M. Coron, "Lyapunov design of stabilizing controllers for cascaded systems," IEEE Trans. Automat. Contr., vol. 36, pp. 1177-1181, Oct. 1991

[17] E. D. Sontag, "Stability and stabilization: Discontinuities and the effect of disturbances," in Nonlinear Analysis, Differential Equations, and Control. Norwell, MA: Kluwer, 1999, pp. 551-598.

[18] G. F. Wredenhagen and P. R. Belanger, "Piecewise-linear LQ control for systems with input constraints," Automatica, vol. 30, pp. 403-416, 1994.

\section{Existence of Carathéodory Solutions in Nonlinear Systems With Discontinuous Switching Feedback Controllers}

\author{
Seung-Jean Kim and In-Joong Ha
}

\begin{abstract}
In this note, we consider the existence of a Carathéodory solution in a single-input-single-output nonlinear system with a discontinuous switching feedback controller. The main contribution is to show that if the nonlinear system can be transformed into a global normal form, then we can specify the value of the discontinuous switching feedback controller on the switching hypersurface so that the closed-loop system has a Carathéodory solution.
\end{abstract}

Index Terms-Differential inclusions, existence of solutions, variable structure systems.

\section{INTRODUCTION}

Discontinuous switching nonlinearities are inherent in many control systems such as adaptive control systems with discontinuous switching laws [4], mechanical systems with friction [5], [6], and variable struc-

Manuscript received May 2, 2002; revised April 29, 2003 and February 8, 2004. Recommended by Associate Editor Z. Lin. The work of S.-J. Kim was supported by the Postdoctoral Fellowship Program of the Korea Science and Engineering Foundation (KOSEF).

S.-J. Kim is with the Information Systems Laboratory, the Department of Electrical Engineering, Stanford University, Stanford, CA 94305 USA (e-mail: sjkim@snu.ac.kr).

I.-J. Ha is with School of Electrical Engineering, Seoul National University, Seoul 151-742, Korea (e-mail: ijha@snu.ac.kr).

Digital Object Identifier 10.1109/TAC.2004.831127 ture control systems [7]-[11]. As a result, the classical existence theorem-which guarantees the existence of Carathéodory (classical) solutions in differential equations with continuous right-hand sides - cannot be applied to the differential equations that govern their dynamic behavior.

Until now, there has been a considerable amount of research work on the solution concepts for differential equations with discontinuous right-hand sides; see, e.g., [1]-[3], [19], [21], [22]. Specifically, Filippov showed that if the discontinuous right-hand sides are replaced by certain set-valued maps, then the resulting differential inclusions, namely, the Filippov differential inclusions always have solutions in a certain sense, which are commonly called Filippov solutions of the original differential equations [1], [2]. In fact, the stability analysis of nonlinear systems with discontinuous switching feedback controllers has been carried out on the basis of the Filippov differential inclusions [4], [7]-[16]. Nonetheless, Filippov solutions do not necessarily characterize their dynamic behavior in a strict sense, since the corresponding set-valued maps are not single-valued but multivalued on the switching hypersurfaces.

In this context, many authors have attempted to find explicit conditions for the existence of Carathéodory solutions in nonlinear systems with discontinuous switching feedback controllers [4], [5]. For instance, it was shown in [4] that, in a special class of adaptive control systems with discontinuous switching laws, the existence of Carathéodory solutions can be guaranteed by appropriately specifying the value of the discontinuous switching laws on the switching hypersurfaces.

In this note, we consider the existence of a Carathéodory solution in a single-input-single-output (SISO) nonlinear system with a discontinuous switching feedback controller. The main contribution is to show that if the nonlinear system can be transformed into a global normal form [20], then we can specify the value of the discontinuous switching feedback controller on the switching hypersurface so that the closed-loop system has a Carathéodory solution.

\section{PRELIMINARIES}

We introduce some definitions and notations used throughout the note. The Euclidean norm of a vector $x$ in $\mathbf{R}^{n}$ is denoted by $\|x\|$. The set of all subsets of a set $X$ is denoted by $2^{X}$. A set-valued map $F$ : $X \rightarrow 2^{Y}$ is a function that associates to any $x \in X$ a subset $F(x)$ of $Y$. The convex hull, $\operatorname{conv}(W)$, of a set $W \subset \mathbf{R}^{n}$ is the smallest convex set that contains $W$. In particular when $W=\left\{x_{1}, x_{2}, \ldots, x_{p}\right\}$, we write $\operatorname{conv}\left(\left\{x_{1}, x_{2}, \ldots, x_{p}\right\}\right)$ simply as $\operatorname{conv}\left(x_{1}, x_{2}, \ldots, x_{p}\right)$. The derivative of a vector-valued function $T: \mathbf{R}^{n} \rightarrow \mathbf{R}^{m}$ at $x$ is denoted by $D T(x) \in \mathbf{R}^{m \times n}$, while the successive Lie derivatives of a scalar function $h: \mathbf{R}^{n} \rightarrow \mathbf{R}$ along a vector-valued function $f: \mathbf{R}^{n} \rightarrow \mathbf{R}^{n}$ are denoted by $L_{f}^{i+1} h \triangleq L_{f} L_{f}^{i} h, i=1,2, \ldots$, where $L_{f}^{1} h(x)=L_{f} h(x)=D h(x) f(x)$. The $i$ th component of a vector-valued function (or a vector) $\xi$ is denoted by $\xi_{i}$.

We consider a SISO nonlinear system of the following form:

$$
\Sigma:\left\{\begin{array}{l}
\dot{x}=f(x)+g(x) u \\
y=s(x),
\end{array} \quad x(t) \in \mathbf{R}^{n}, u(t), y(t) \in \mathbf{R}\right.
$$

where $f$ and $g$ are smooth functions from $\mathbf{R}^{n}$ into $\mathbf{R}^{n}$ and $s$ is a smooth function from $\mathbf{R}^{n}$ into $\mathbf{R}$. Here, we assume that the control input $u$ is given by a discontinuous feedback controller of the form

$$
u=k(x) \triangleq \begin{cases}u_{+}(x), & s(x)>0 \\ u_{-}(x), & s(x)<0\end{cases}
$$

where $s, u_{+}$, and $u_{-}$are continuous functions from $\mathbf{R}^{n}$ into $\mathbf{R}$. As a matter of fact, variable structure control systems [11] as well as adaptive control systems with discontinuous switching laws [4] and me- 